\title{
Review of: "Bulk and nanoparticles of zinc oxide exerted their beneficial effects by conferring modifications in transcription factors, histone deacetylase, carbon and nitrogen assimilation, antioxidant biomarkers, and secondary metabolism in soybean"
}

Radka Podlipná ${ }^{1}$

1 The Czech Academy of Sciences

Potential competing interests: The author(s) declared that no potential competing interests exist.

The text is very well written. I appreciate that the authors used both nano and mass counterpart resp. and two different methods of application. The methods slightly complicated the presentation of the results due to the different uptake of $\mathrm{Zn}$ by roots and leaves. The introduction lacks the composition of the nutrient medium that was used for irrigation. Thus, the improvement in all physiological aspects after all treatments could be due to zinc deficiency in the control plants. The positive effect of zinc NPs has already been described, but a direct comparison with the bulk form is useful.

I suggest to the authors that the abstract be slightly rearranged, which readers who are not specialists in transcriptomics and do not know the name of the genes would not have to understand. In my opinion, sentences similar to those used in the conclusion part would appeal to a wide range of readers.

The heat map needs more comments to bring readers some new knowledge. Everything can be correlated even if there are no connections.

Other remarks have been highlighted in the text. 